\title{
GFR-Specific versus GFR-Agnostic Cutoffs for Parathyroid Hormone and Fibroblast Growth Factor-23 in Advanced Chronic Kidney Disease
}

\author{
Mark Canney ${ }^{\mathrm{a}}$ Ognjenka Djurdjev $^{\mathrm{b}}$ Mila Tang $^{\mathrm{c}}$ Claudia Zierold $^{\mathrm{d}}$ \\ Frank Blockid Myles Wolfe Adeera Levin ${ }^{a}$ \\ ${ }^{a}$ Division of Nephrology, University of British Columbia, Vancouver, BC, Canada; ${ }^{b}$ BC Renal Agency, Analytics, \\ Vancouver, BC, Canada; ' Nephrology Research, St. Paul's Hospital, Vancouver, BC, Canada; d Diasorin Inc., Scientific \\ Affairs, Stillwater, MN, USA; ${ }^{e}$ Division of Nephrology, Department of Medicine, Duke University School of Medicine, \\ and Duke Clinical Research Institute, Durham, NC, USA
}

\author{
Keywords \\ Chronic kidney disease - Fibroblast growth factor 23 . \\ Glomerular filtration rate $\cdot$ Outcomes $\cdot$ Parathyroid \\ hormone
}

\begin{abstract}
Background: In the majority of patients with advanced chronic kidney disease (CKD), values of parathyroid hormone (PTH1-84) and fibroblast growth factor 23 (FGF-23) exceed the normal reference range, potentially as an appropriate adaptation to reduced glomerular filtration rate (GFR). We tested whether GFR-specific cutoffs for PTH1-84 and FGF-23 could better identify patients with inappropriately high PTH1-84 and FGF-23 for their degree of CKD and thereby improve prognostication of clinical outcomes compared to a uniform threshold. Methods: Prospective pan-Canadian cohort of 1,812 patients with mean estimated GFR (eGFR) $28.9 \mathrm{~mL} / \mathrm{min} / 1.73 \mathrm{~m}^{2}$ followed for a median of 52 months. Repeated log-rank tests were used to identify optimal cutoffs for PTH1-84 and FGF-23 within eGFR strata $(<20,20-29$ and $\geq 30 \mathrm{~mL} / \mathrm{min} / 1.73 \mathrm{~m}^{2}$ ) that maximally differentiated
\end{abstract}

high- and low-risk populations for (1) cardiovascular (CV) events (fatal or nonfatal myocardial infarction, coronary revascularization, stroke, heart failure) and (2) renal events (initiation of chronic renal replacement therapy). In multivariable models, we examined the association between GFR-specific cutoffs and outcomes and compared their added prognostic value to existing uniform thresholds. Results: Risk-based cutoffs for PTH1-84 and FGF-23 increased in a graded fashion with decreasing eGFR. Among patients with eGFR $<20 \mathrm{~mL} / \mathrm{min} / 1.73 \mathrm{~m}^{2}, \mathrm{CV}$ risk-based cutoffs for PTH184 and FGF-23 were 3.4 and 5.5 times the upper limit of normal, respectively, and reclassified 31.9 and $35.1 \%$ of patients when added to a multivariable base model for CV events. In contrast, the addition of PTH1-84 and FGF-23 to the base model using uniform cutoffs failed to reclassify such patients. Similar findings were demonstrated for renal outcomes. Conclusion: GFR-specific risk-based cutoffs for PTH1-84 and FGF-23 may facilitate more meaningful risk stratification in advanced CKD than current GFR-agnostic reference ranges derived from healthy adults. This may be most applicable in those with severely reduced GFR.

(c) 2019 The Author(s)

Published by S. Karger AG, Basel

Dr. Adeera Levin

Department of Medicine, University of British Columbia

St. Paul's Hospital, 1081 Burrard Street Room 6010A

Vancouver, BC V6Z1Y6 (Canada)

E-Mail alevin@ providencehealth.bc.ca
This article is licensed under the Creative Commons AttributionNonCommercial-NoDerivatives 4.0 International License (CC BYNC-ND) (http://www.karger.com/Services/OpenAccessLicense) Usage and distribution for commercial purposes as well as any distribution of modified material requires written permission. 


\section{Introduction}

Within the chronic kidney disease mineral bone disease (CKD-MBD) paradigm, there is increasing recognition of a complex interplay between biochemical and hormonal changes at various levels of glomerular filtration rate (GFR). These processes include vitamin D deficiency, disordered phosphate homeostasis, elevated fibroblast growth factor 23 (FGF-23), and secondary hyperparathyroidism, all of which contribute to bone and vascular health [1-5]. Abnormal levels of parathyroid hormone (PTH) and FGF-23 have consistently been associated with adverse clinical outcomes [6-8]. Although both biomarkers are inversely correlated with GFR levels, current reference values for PTH, and FGF-23 are based on distributions observed in healthy adults in the general population and are fixed across the GFR spectrum. These thresholds tend to identify the majority of patients with advanced CKD as "abnormal." This is problematic because not all of these patients are at higher risk of adverse outcomes, with the result that uniform thresholds for PTH and FGF-23 have limited capacity for risk-discrimination in this population.

For some patients, elevations in PTH or FGF-23 could represent an adaptive physiological response rather than an exaggerated response relative to the severity of their CKD that connotes increased risk. The 2017 Kidney Disease: Improving Global Outcomes guidelines [9] acknowledge that increases in PTH could reflect compensatory responses that are appropriate in the context of reduced GFR. Inherent in this interpretation is that, at a given level of GFR, there is a threshold value of PTH which, although above the normal reference range, might be considered "tolerable." A key unanswered question is where along the range of PTH or FGF-23 a result changes from a value that is expected for a given level of kidney function to a value that confers higher risk of important outcomes. This conceptual threshold thus has 2 defining characteristics. The first is that it is specific to an individual's level of kidney function. The second is that its identification will improve risk stratification for that individual. While intuitive, this concept has not been formally evaluated in patients with advanced CKD. Refining the interpretation of PTH and FGF-23 in this way could help resolve some of the clinical confusion that currently surrounds these biomarkers and generate hypotheses for future studies.

We tested whether GFR-specific cutoffs for PTH1-84 and FGF-23 could better identify patients with inappropriately high PTH1-84 and FGF-23 for their degree of
CKD and thereby improve prognostication of cardiovascular $(\mathrm{CV})$ and renal outcomes compared to a uniform GFR-agnostic threshold.

\section{Materials and Methods}

\section{Design, Setting, and Participants}

This was a prospective observational study from the Canadian study of Prediction of Dialysis, Death, and Interim CV events (CanPREDDICT), a pan-Canadian cohort of 2,544 patients under the care of nephrologists from 2008 to 2013 inclusive [10]. Eligible participants had an estimated GFR (eGFR) of $15-45 \mathrm{~mL} /$ $\mathrm{min} / 1.73 \mathrm{~m}^{2}$ and provided informed consent. Those excluded had a life expectancy $<12$ months, organ transplant(s), or active vasculitis. Participants were followed for 3 years at 6 -monthly intervals and annually thereafter. The present analysis includes 1,812 participants who had complete data for PTH1-84 and FGF-23 measured at the 6-month visit, and who had not progressed to dialysis by that time (online suppl. Fig. S1; for all online suppl. material, see www.karger.com/doi/10.1159/000501189). Among included patients, $92 \%$ had an eGFR between 15 and $45 \mathrm{~mL} / \mathrm{min} / 1.73 \mathrm{~m}^{2}$ ( $6 \%$ of patients had an eGFR $>45 \mathrm{~mL} / \mathrm{min} / 1.73 \mathrm{~m}^{2}$ and $2 \%$ had an eGFR $<15 \mathrm{~mL} / \mathrm{min} / 1.73 \mathrm{~m}^{2}$ ). CanPREDDICT was registered at www.clinicaltrials.gov (NCT00826319).

\section{Outcomes}

The outcomes used to define risk-based cutoffs were initiation of chronic renal replacement therapy and cardiovascular events. All events were centrally adjudicated based on source documentation by a panel of clinicians including a cardiologist, nephrologist, and neurologist. We defined a CV event as one of the following: fatal or nonfatal myocardial infarction, coronary revascularization (coronary artery bypass graft, percutaneous coronary intervention, percutaneous transluminal coronary angioplasty), stroke, or congestive heart failure (defined as dyspnea plus 2 of: bibasilar rales, raised jugular venous pressure or pulmonary edema on chest X-ray).

\section{Exposure}

Measurement of bone mineral biomarkers was performed in a central accredited laboratory on a DiaSorin LIAISON ${ }^{\circledR}$ XL analyzer using fully-automated chemiluminescent immunoassays for PTH1-84 (\#310630) and intact FGF-23 (\#318700). The PTH assay is a 3rd generation assay which measures the biologically active PTH(1-84) whole peptide, enabling standardization and consistent measurement [11], unlike commonly used and inconsistent intact PTH assays (iPTH or 2nd generation) that also detect inactive PTH(7-84) [12-14]. The FGF-23 assay detects nonfragmented protein and is standardized to a full-length purified recombinant protein to ensure assay accuracy [15]. The upper limit of normal (ULN) for the PTH1-84 assay of $39.4 \mathrm{pg} / \mathrm{mL}$ was defined as the 97.5 th centile from the distribution of 125 healthy adults with 25 -hydroxyvitamin D levels $>30 \mathrm{ng} / \mathrm{mL}$. The ULN for the FGF-23 assay of $95.4 \mathrm{pg} / \mathrm{mL}$ was defined as the 97.5 th centile from the distribution of 910 healthy adults with eGFR $>60 \mathrm{~mL} / \mathrm{min} / 1.73 \mathrm{~m}^{2}$.

\section{Covariates}

Demographic variables included age, sex, and race. Clinical variables, including diabetes mellitus, ischemic heart disease, congestive heart failure, medication use, height, weight and blood 
pressure (BP), were collected at the 6-month study visit to align with the measurements of PTH1-84 and FGF-23. Creatinine, hemoglobin, urinary albumin to creatinine ratio, bicarbonate, albumin, and phosphate were measured in local accredited laboratories using standardized assays. Laboratory variables measured closest to the 6-month visit were used. GFR was estimated from creatinine using the CKD Epidemiology Collaboration equation [16].

\section{Statistical Analysis}

Analyses were performed using SAS version 9.3 (SAS Institute Inc., Cary, NC, USA). Continuous variables are presented as means (SD) or medians (interquartile range) as appropriate. Categorical variables are presented as count (percent). We grouped participants into categories of eGFR: $<20,20-29, \geq 30 \mathrm{~mL} / \mathrm{min} / 1.73 \mathrm{~m}^{2}$. These categories were chosen to be consistent with clinical staging of eGFR, while also recognizing the heterogeneity among patients with eGFR values between 15 and $30 \mathrm{~mL} / \mathrm{min} / 1.73 \mathrm{~m}^{2}$. We compared participant characteristics among eGFR categories using ANOVA or the Kruskal-Wallis test for continuous variables and the chi-square test for categorical variables. Variables with skewed distributions were natural log transformed for analysis.

To confirm their association with each outcome, PTH1-84 and FGF-23 were initially included as continuous predictors (logtransformed) in multivariable Cox proportional hazards models. We considered the following covariates for selection in the model based on prior literature: age, sex, diabetes, preexisting cardiac disease, nonsteroidal anti-inflammatory drugs, beta-blockers, angiotensin-converting enzyme inhibitors, BP, weight, eGFR, albumin to creatinine ratio, hemoglobin, albumin, potassium, bicarbonate, calcium, and phosphate. For each outcome, we employed a backward selection regression strategy to select covariates in the model based on a threshold $p$ value of $\leq 0.1$. Routinely collected laboratory variables were retained in the model for renal events to be consistent with the Kidney Failure Risk Equation [17].

Risk-based cutoffs for PTH1-84 and FGF-23 within each eGFR category were determined using an outcome-based approach [18, 19]. We defined as an optimal cut point the value of the continuous biomarker that, unadjusted for other covariates, best separated high- and low-risk participants for each outcome. Several potential cut points were tested along the range of each biomarker using the log-rank statistic, with adjustment of $p$ values for multiple testing, using the approach proposed by Contal and O'Quigley [20]. Visually, these cut points were confirmed by inspecting the functional form of Martingale residuals. Within each eGFR category, we compared the prognostic value of uniform cutoffs (categorical PTH184 and FGF-23 dichotomized using the ULN) and risk-based cutoffs (categorical PTH1-84 and FGF-23 dichotomized using GFRspecific cutoffs) when added to a multivariable base model. Metrics of performance included category-free net reclassification index, area under the receiver operating characteristic curve, and integrated discrimination improvement [21-24].

\section{Results}

\section{Characteristics of the Study Population}

Table 1 describes the characteristics of the cohort, overall and by eGFR category. Mean (SD) age was 68.9 (12.6) years, $62 \%$ were male and $90 \%$ were Caucasian. Mean (SD) eGFR was $28.9(10.0) \mathrm{mL} / \mathrm{min} / 1.73 \mathrm{~m}^{2}$ and $19.4,40.3$, and $40.3 \%$ of participantshad an eGFR $<20,20-29$ and $\geq 30 \mathrm{~mL} /$ $\mathrm{min} / 1.73 \mathrm{~m}^{2}$, respectively. The proportion of participants prescribed vitamin $\mathrm{D}$, phosphate binders, and calcium supplements was 36,25 , and $27 \%$, respectively.

\section{Distribution of PTH1-84 and FGF-23}

The majority of participants (80\%) had a value of PTH184 above the ULN $(>39.4 \mathrm{pg} / \mathrm{mL})$. Among participants with eGFR $<20 \mathrm{~mL} / \mathrm{min} / 1.73 \mathrm{~m}^{2}$, median PTH1-84 was twice the ULN (online suppl. Fig. S2). Similarly, participants with eGFR $<20 \mathrm{~mL} / \mathrm{min} / 1.73 \mathrm{~m}^{2}$ had a median FGF-23 more than twice the ULN (210 vs. $95.4 \mathrm{pg} / \mathrm{mL}$ ). In a subgroup analysis, we examined the distributions of PTH1-84 and FGF-23 in participants who were receiving and not receiving medications known to influence their concentrations (online suppl. Table S1). Participants receiving vitamin D had nonsignificantly lower PTH1-84 values and higher FGF-23 values compared to untreated participants.

\section{Association between PTH1-84 and FGF-23 and Outcomes}

There were 551 (30.4\%) CV and renal events recorded during a median (interquartile range) follow-up time of 52 (29-54) months, representing 6,244 person-years at risk: $341(18.8 \%)$ participants experienced a first CV event resulting in 59.1 (95\% CI 53.2-65.6) events per 1,000 personyears; and 307 (16.9\%) participants experienced a first renal event resulting in 53.0 (95\% CI 47.4-59.3) events per 1,000 person-years. A total of 97 (5.4\%) participants had both a CV and renal event, the majority of whom $(n=65$, 67\%) experienced a CV event prior to their renal event. The relationship between continuous PTH1-84 and FGF23 and each outcome is detailed in online supplementary Table S2. Each SD increase in PTH1-84 (log-transformed) was independently associated with an $11 \%$ increased risk of CV events and a $23 \%$ increased risk of renal events. The corresponding risk estimates for FGF-23 were 21 and 31\%.

\section{GFR-Specific Cutoffs for PTH1-84 and FGF-23}

Since cutoffs rather than continuous values are generally used in clinical practice, we explored optimal cutoffs for both biomarkers. Risk-based cutoffs for PTH1-84 and FGF-23, for each eGFR category, are described in Table 2. These cutoffs were higher for eGFR $<20 \mathrm{~mL} / \mathrm{min} / 1.73 \mathrm{~m}^{2}$ compared to more preserved levels of kidney function. The PTH1-84 cutoff for CV events was 3.4 times the ULN in this group, compared to 2.7 times the ULN for eGFR 20$29 \mathrm{~mL} / \mathrm{min} / 1.73 \mathrm{~m}^{2}$ and 1.2 times the ULN for eGFR $\geq 30$ 
Table 1. Characteristics of the study cohort by levels of eGFR

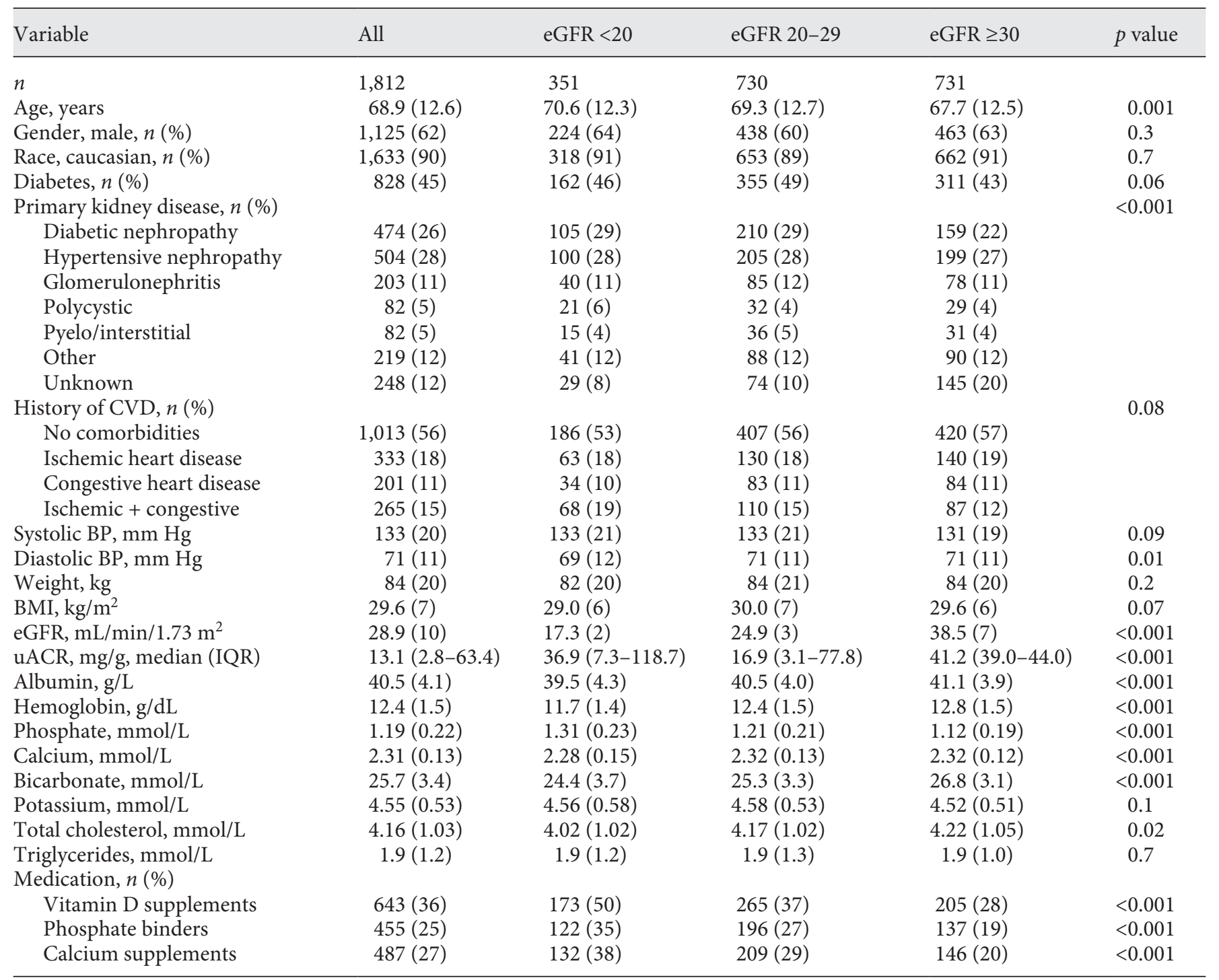

Among patients who were prescribed vitamin $\mathrm{D}$, the majority (59.8\%) were receiving a nutritional supplement compared to an active sterol such as alfacalcidol (30.8\%) or calcitriol (9.4\%).

$\mathrm{BMI}$, body mass index; BP, blood pressure; CVD, cardiovascular disease; eGFR, estimated glomerular filtration rate; uACR, urinary albumin to creatinine ratio; IQR, interquartile range.

$\mathrm{mL} / \mathrm{min} / 1.73 \mathrm{~m}^{2}$. The PTH1-84 cutoffs for renal events were comparatively lower than those for $\mathrm{CV}$ events across the GFR range. Nonetheless, among participants with eGFR $<20 \mathrm{~mL} / \mathrm{min} / 1.73 \mathrm{~m}^{2}$, the cutoff for renal events was 2.4 times the ULN. The pattern for FGF-23 cutoffs was similar to that for PTH1-84: we observed a graded increase in FGF-23 cutoff values for each outcome with decreasing levels of eGFR, and FGF-23 cutoff values were higher for $\mathrm{CV}$ events compared to renal events.
Figure 1 illustrates the distribution of participants above and below each type of cutoff for both biomarkers. It also demonstrates, at each level of eGFR, the proportion of participants who had biomarker values between the uniform cutoff and the risk-based cutoff. For CV events, $61 \%$ of participants with eGFR $<20 \mathrm{~mL} / \mathrm{min} / 1.73 \mathrm{~m}^{2}$ had a PTH1-84 value above the uniform cutoff but below their GFR-specific cutoff. This proportion became smaller at higher levels of eGFR, consistent with the risk-based 
Table 2. GFR- and outcome-specific cut-offs for PTH1-84 and FGF-23. For each risk-based cutoff, the corresponding maximum Q statistic and $\log$-rank $p$ value are provided

\begin{tabular}{|c|c|c|c|c|c|c|c|c|c|c|}
\hline Biomarker & Cutoff & \multicolumn{3}{|c|}{$\mathrm{eGFR}<20 \mathrm{~mL} / \mathrm{min} / 1.73 \mathrm{~m}^{2}$} & \multicolumn{3}{|c|}{ eGFR $20-29 \mathrm{~mL} / \mathrm{min} / 1.73 \mathrm{~m}^{2}$} & \multicolumn{3}{|c|}{$\mathrm{eGFR} \geq 30 \mathrm{~mL} / \mathrm{min} / 1.73 \mathrm{~m}^{2}$} \\
\hline \multirow{2}{*}{$\begin{array}{l}\text { PTH1-84, } \\
\mathrm{pg} / \mathrm{mL}\end{array}$} & CV event risk-based & 134.3 & 1.41 & 0.038 & 104.6 & 0.99 & 0.30 & 42.9 & 1.12 & 0.16 \\
\hline & RRT risk-based & 93.7 & 2.08 & 0.0003 & 49.4 & 2.12 & 0.0003 & 53.5 & 1.70 & 0.006 \\
\hline \multirow{2}{*}{$\begin{array}{l}\text { FGF-23 } \\
\text { (intact), } \\
\mathrm{pg} / \mathrm{mL}\end{array}$} & Uniform & 95.0 & $\mathrm{n} / \mathrm{a}$ & $\mathrm{n} / \mathrm{a}$ & 95.0 & $\mathrm{n} / \mathrm{a}$ & $\mathrm{n} / \mathrm{a}$ & 95.0 & $\mathrm{n} / \mathrm{a}$ & $\mathrm{n} / \mathrm{a}$ \\
\hline & RRT risk-based & 311.1 & 2.85 & $<0.0001$ & 192.5 & 2.83 & $<0.0001$ & 141.2 & 1.86 & 0.002 \\
\hline
\end{tabular}

CV, cardiovascular; RRT, renal replacement therapy; GFR, glomerular filtration rate; eGFR, estimated GFR; PTH, parathyroid hormone; FGF-23, fibroblast growth factor 23; n/a, not applicable.

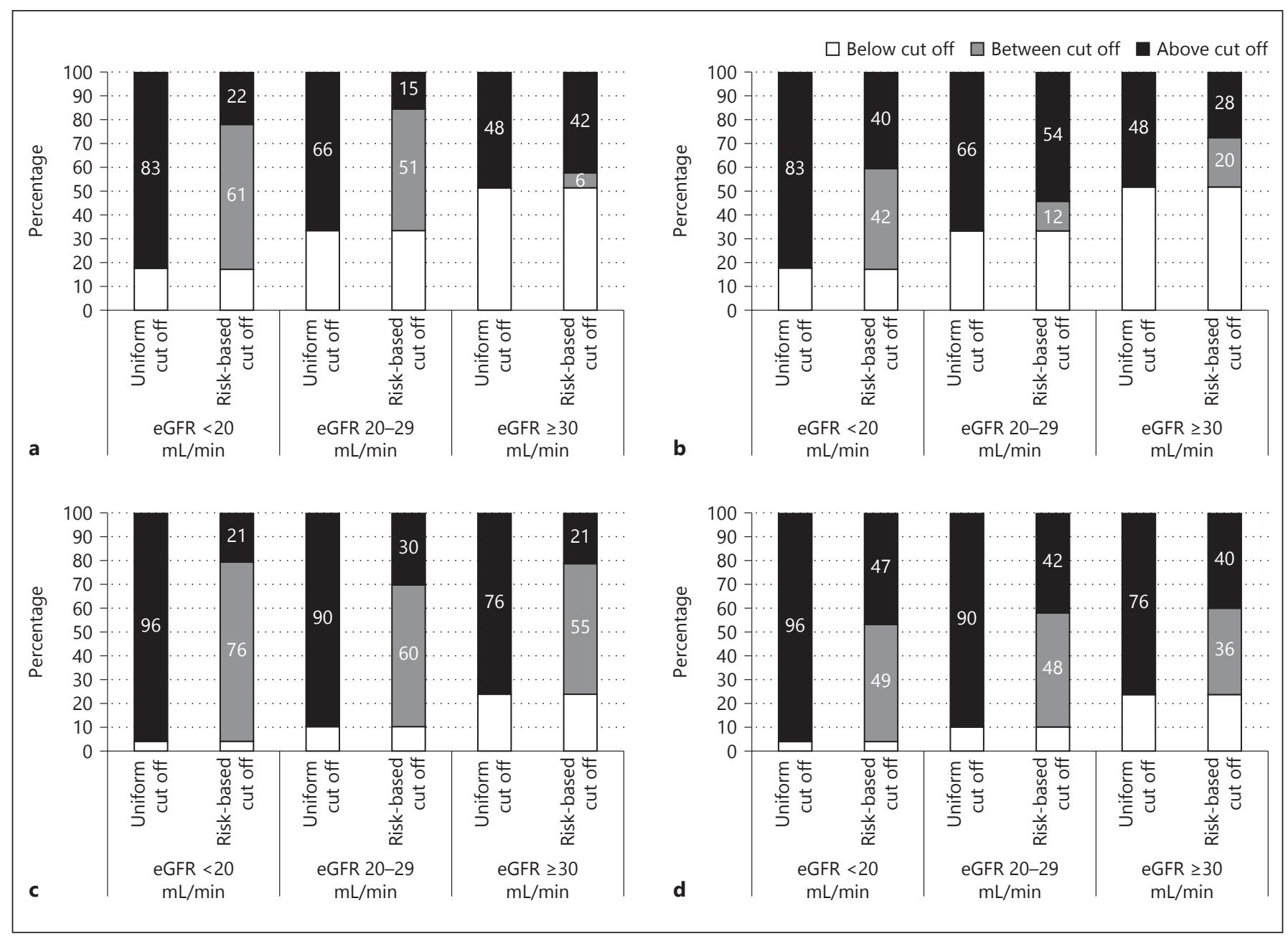

Fig. 1. Distribution of participants above and below uniform and risk-based cutoffs by category of eGFR: (a) PTH1-84 and cardiovascular events, (b) PTH1-84 and renal events, (c) FGF-23 and cardiovascular events, (d) FGF-23 and renal events. eGFR, estimated glomerular filtration rate. 
Table 3. Net reclassification index (95\% CI) for biomarker cutoff values for CV and renal events

\begin{tabular}{|c|c|c|c|c|c|c|}
\hline \multirow[b]{2}{*}{$\begin{array}{l}\text { PTH } 1-84 \text { cutoff values for } \\
\text { CV event risk, } \mathrm{pg} / \mathrm{mL}\end{array}$} & \multicolumn{2}{|c|}{$\mathrm{eGFR}<20 \mathrm{~mL} / \mathrm{min} / 1.73 \mathrm{~m}^{2}$} & \multicolumn{2}{|c|}{ eGFR $20-29 \mathrm{~mL} / \mathrm{min} / 1.73 \mathrm{~m}^{2}$} & \multicolumn{2}{|c|}{$\mathrm{eGFR} \geq 30 \mathrm{~mL} / \mathrm{min} / 1.73 \mathrm{~m}^{2}$} \\
\hline & GFR-agnostic 39.4 & GFR-specific 134.3 & GFR-agnostic 39.4 & GFR-specific 104.6 & GFR-agnostic 39.4 & GFR-specific 42.9 \\
\hline $\begin{array}{l}\text { Unadjusted model: GFR-specific vs. } \\
\text { GFR-agnostic }\end{array}$ & $17.7(10.8$ to 25.0$)$ & & $15.3(-14.1$ to 16.4$)$ & & $25.0(9.3$ to 37.2$)$ & \\
\hline Base model ${ }^{1}$ vs.base model + PTH $1-84$ & $9.7(-12.5$ to 28.1$)$ & $31.9(23.4$ to 48.8$)$ & $1.2(-1.4$ to 15.4$)$ & $7.9(-5.8$ to 25.5$)$ & $-12.1(-4.1$ to 24.5$)$ & $21.3(-3.6$ to 36.0$)$ \\
\hline $\begin{array}{l}\text { PTH } 1-84 \text { cutoff values for } \\
\text { renal event risk, } \mathrm{pg} / \mathrm{mL}\end{array}$ & GFR-agnostic 39.4 & GFR-specific 93.7 & GFR-agnostic 39.4 & RRT risk cutoff 49.4 & GFR-agnostic 39.4 & GFR-specific 53.5 \\
\hline $\begin{array}{l}\text { Unadjusted model: GFR-specific vs. } \\
\text { GFR-agnostic }\end{array}$ & $21.3(-7.3$ to 50.6$)$ & & $37.3(5.4$ to 55.9$)$ & & $11.9(-21.0$ to 66.8$)$ & \\
\hline Base model $^{2}$ vs. base model + PTH1-84 & $15.7(-7.2$ to 29.1$)$ & $39.4(-17.0$ to 69.9$)$ & $26.7(20.1$ to 32.9$)$ & $37.3(-32.0$ to 59.2$)$ & $40.7(-11.3$ to 76.1$)$ & $37.3(-3.9$ to 71.9$)$ \\
\hline $\begin{array}{l}\text { FGF- } 23 \text { cutoff values for } \\
\text { CV event risk, } \mathrm{pg} / \mathrm{mL}\end{array}$ & GFR-agnostic 95.4 & GFR-specific 528.5 & GFR-agnostic 95.4 & GFR-specific 228.1 & GFR-agnostic 95.4 & GFR-specific 177.7 \\
\hline $\begin{array}{l}\text { Unadjusted model: GFR-specific vs. } \\
\text { GFR-agnostic }\end{array}$ & 22.8 (1.1 to 43.7$)$ & & $20.6(5.6$ to 42.3$)$ & & $21.4(-6.0$ to 43.4$)$ & \\
\hline Base model $^{1}$ vs. base model + FGF-23 & $-3.0(-20.5$ to 21.2$)$ & $35.1(10.2$ to 48.1$)$ & $-3.0(-17.7$ to 20.1$)$ & $23.1(3.5$ to 45.6$)$ & $10.6(-9.9$ to 21.8$)$ & $31.8(11.8$ to 48.5$)$ \\
\hline $\begin{array}{l}\text { FGF- } 23 \text { cutoff values for } \\
\text { renal event risk, } \mathrm{pg} / \mathrm{mL}\end{array}$ & GFR-agnostic 95.4 & GFR-specific 311.1 & GFR-agnostic 95.4 & GFR-specific 192.5 & GFR-agnostic 95.4 & GFR-specific 141.2 \\
\hline $\begin{array}{l}\text { Unadjusted model: GFR-specific vs. } \\
\text { GFR-agnostic }\end{array}$ & 46.7 (27.4 to 82.2 ) & & $24.9(0.8$ to 48.5$)$ & & $25.2(3.3$ to 99.2$)$ & \\
\hline Base model $^{2}$ vs. base model + FGF-23 & $-17.5(-45.6$ to 28.1$)$ & $56.8(32.7$ to 77.2$)$ & $2.8(-34.1$ to 26.0$)$ & $42.9(19.9$ to 63.3$)$ & $9.2(-23.5$ to 38.8$)$ & $61.7(-40.7$ to 84.5$)$ \\
\hline
\end{tabular}

cutoff approaching the ULN. Similarly, with decreasing eGFR, a greater proportion of participants had FGF-23 values above the uniform cutoff but below the risk-based cutoff (Fig. 1). In multivariable models, participants with PTH1-84 and FGF-23 values above the uniform cutoff but below their GFR-specific cutoff had similar risks of $\mathrm{CV}$ and renal events compared to participants whose biomarker values were within the normal reference range (online suppl. Table S3).

\section{GFR-Specific Cutoffs and Outcomes}

Table 3 shows the reclassification associated with riskbased and uniform cutoffs of PTH1-84 and FGF-23 within eGFR categories. In unadjusted analyses, directly comparing the 2 types of cutoff, GFR-specific cutoffs demonstrated superior performance for $\mathrm{CV}$ and renal outcomes across all categories of eGFR. Among patients with eGFR $<20 \mathrm{~mL} / \mathrm{min} / 1.73 \mathrm{~m}^{2}$, GFR-specific cutoffs for FGF-23 appropriately reclassified 22.8 and $46.7 \%$ of patients for $\mathrm{CV}$ and renal outcomes, respectively. For CV outcomes, the net reclassification index was driven mainly by appropriate reclassification of patients who did not experience an event as lower risk (online suppl. Table S4). In a multivariable context, GFR-specific cutoffs for PTH1-84 and FGF-23 substantially improved reclassification for both outcomes. In contrast, the addition of uniform cutoffs for PTH1-84 and FGF-23 to the base model resulted in either no improvement or only modest improvements in reclassification. Similar patterns were observed for differences in area under the receiver operating characteristic curve and integrated discrimination improvement (online suppl. Table S5, S6). In general, improvements associated with GFR-specific cutoffs were more consistently demonstrated for FGF-23 compared to PTH1-84.

\section{Discussion}

In this large cohort of patients with advanced CKD, elevations in PTH1-84 and FGF-23 were associated with higher risk of CV and renal events, over and above traditional and CKD-specific risk factors. Cutoff values for PTH1-84 and FGF-23 that maximally differentiated risk 
of $\mathrm{CV}$ and renal events were progressively higher with decreasing eGFR and demonstrated improved prognostication of each outcome compared to uniform cutoffs. Individuals with biomarker values above the normal range but below their GFR-specific cutoff had a similar risk profile to those individuals whose biomarker values were within the normal reference range. Collectively, these findings support the notion that GFR-specific cutoffs for PTH1-84 and FGF-23, underpinned by risk of important clinical outcomes, can be identified and could provide clinicians with a more meaningful interpretation of these biomarkers than existing reference ranges that are derived from healthy adults and fixed across the GFR spectrum.

It has long been recognized that the concentrations of PTH1-84 and FGF-23 increase as kidney function declines. Numerous studies have demonstrated that higher values of these biomarkers are associated with increased risk of clinical outcomes $[6-8,25,26]$. The challenge for clinicians is in differentiating elevations in PTH1-84 or FGF-23 that are attributable to declining GFR, and therefore an "expected" physiological or adaptive response, from excessively elevated values that signal an aberration beyond that expected for GFR decline, thus warranting extra attention. Outcome-oriented approaches have been used to establish optimal cut points in other continuous covariates such as ambulatory BP and cancer biomarkers $[27,28]$. The rationale for their use in the present analysis was to optimally risk stratify within the advanced CKD population, in order to identify individuals whose biomarker values exceeded the risk-based threshold specific to their level of GFR. To our knowledge, this concept has not been formally tested in this way for CKD-MBD markers. We used outcomes that are important to both clinicians and patients to establish these risk thresholds. Whether or not treatment of the laboratory abnormality will change these outcomes is outside the scope of this study. This analysis did however establish that it is possible to derive GFR-specific thresholds of PTH1-84 and FGF-23 that discriminate risk of CV and renal outcomes within a well-characterized population with CKD. Among patients with more advanced disease (eGFR $<20 \mathrm{~mL}$ / $\min / 1.73 \mathrm{~m}^{2}$ ), these thresholds are considerably higher than existing uniform thresholds, which would inevitably result in a trade-off of greater specificity for lower sensitivity. In this context, there is no perfect way to test the usefulness of a biomarker. We therefore evaluated the performance of GFR-specific cutoffs using a combination of metrics, all of which demonstrated consistent results. Maintaining the status quo of current GFR-agnostic cut- offs is unlikely to advance our understanding of risks associated with PTH1-84 and FGF-23, given our results which emphasize their limited utility for risk stratification in CKD patients.

We specifically investigated PTH because, although measuring PTH is part of usual care in CKD, its clinical importance as a biomarker is poorly understood. The 2017 Kidney Disease: Improving Global Outcomes guidelines [9] state: "In patients with CKD G3a-G5 not on dialysis, the optimal PTH level is not known." Without an accepted level to target, clinicians are left wondering whether or not PTH should be treated and, by extension, if PTH should even be measured. Using the methodology described herein, it may be possible to better inform clinical interpretation of PTH in these patients. It is expected that PTH will be elevated among patients with GFR $<20 \mathrm{~mL} / \mathrm{min} / 1.73 \mathrm{~m}^{2}$, and therefore the threshold at which PTH becomes a harbinger of risk is likely to be higher than the upper limit of a reference range drawn from healthy individuals. We identified this threshold to be 3.4 times the ULN for CV events, and 2.4 times the ULN for renal events. Conversely, among individuals with more preserved kidney function (GFR $>30 \mathrm{~mL}$ / $\min / 1.73 \mathrm{~m}^{2}$ ), the corresponding risk thresholds were closer to the normal reference range, in keeping with the distribution of PTH in that subpopulation. Studies of surrogate $\mathrm{CV}$ outcomes are consistent with our findings. In a sample of 1,152 CKD patients without prior history of $\mathrm{CV}$ disease, a PTH $>110 \mathrm{pg} / \mathrm{mL}$ was identified as an independent predictor of accelerated progression of common carotid intima-media thickness [29]. We were also interested in FGF-23 because although several studies have identified FGF-23 as a strong predictor of adverse outcomes in CKD $[6,7]$, possibly mediated by cardiac toxicity [30], FGF-23 has not yet gained traction in clinical practice. Clinical trials have not conclusively demonstrated that interventions are effective at lowering FGF-23 levels $[31,32]$. Our data indicate that optimal risk-based thresholds for FGF-23 increase as GFR declines, and the thresholds for CV events are different to the thresholds for renal events. By delineating risk by GFR status and specific clinical outcomes, this approach could provide a means of refining selective exclusion criteria to enrich clinical trials with higher risk participants who may benefit from treatment targeted at lowering FGF-23. While it was beyond the intention of this paper to develop a risk prediction model, future studies may want to explore the development and validation of risk prediction scores in CKD patients using GFR-specific cutoffs for PTH and FGF-23, alone or in combination. 
A key strength of the change-point model is the identification of an optimal cut point based on outcomes, as opposed to arbitrarily categorizing the exposure $[33,34]$. The method assumes that an optimal cut point truly exists and will generate 2 distinct groups with respect to risk. This may not be the case, and there could be $>1$ cut point along the distribution of the biomarker. As the cut point is sought using statistical criteria, the risk of type 1 error is inflated [35]. We counteracted this by adjusting the $p$ values for multiple testing. The cut points are dependent on the observed outcomes and may not be generalizable to other cohorts. This is true of other approaches to categorizing continuous exposures such as the use of percentiles, which are specific to the underlying distribution in a sample. Our findings for CV outcomes were more consistent for FGF-23 than PTH1-84, which might reflect the stronger underlying association between FGF-23 and CV disease. For the same reason, an alternative outcome such as bony fracture might arguably be more appropriate for PTH1-84. Additionally, the prognostic importance of PTH and FGF-23 could be different depending on the underlying etiology of kidney disease, for example, among individuals with diabetic kidney disease. We do not propose that the exact cutoffs identified in this analysis are the "right" ones for all situations. Rather, we encourage other investigators to employ this methodological approach in other cohorts in order to validate our findings and further refine this concept.

Our study design has some strengths and limitations. As in all observational cohorts, there may have been measured or unmeasured confounding to explain the results. Data for other bone mineral markers such as alkaline phosphatase were not collected universally in study participants, which may have informed our findings. The sample was $90 \%$ Caucasian, limiting generalizability of the findings to other racial or ethnic groups. Strengths of our design include the capture of adjudicated outcome events over a reasonably long follow-up period of 5 years. We leveraged data from the population of interest, that is, patients with CKD receiving contemporary nephrology care. The sample included a large number of patients with moderate to advanced disease (eGFR $<30 \mathrm{~mL} / \mathrm{min} / 1.73 \mathrm{~m}^{2}$ ), the subgroup in which interventions for CKD-MBD are most frequently contemplated.

This study is a first attempt using state-of-the-art diagnostic tests and an outcome-based approach to identify GFR-specific thresholds of PTH1-84 and FGF-23. The identification of GFR-specific cutoffs could offer a more meaningful interpretation of these biomarkers in advanced CKD. We suggest that future studies validate our findings with the aim of developing a set of GFR-specific values for laboratory tests using larger and more diverse CKD populations. Furthermore, adopting a more refined approach to the interpretation of PTH1-84 and FGF-23, informed by both the level of GFR and the risk of specific clinical outcomes, could facilitate more appropriate design of future clinical trials.

\section{Acknowledgments}

We thank all of the patients and their physicians for participating in the study. We thank all the CanPREDDICT investigators and coordinators for data and sample collection: Mohsen Agharazii, MD, Joanne Blouin, France Samson, CHUQ: L'Hôtel-Dieu de Québec; Ayub Akbarii, MD, Judy Cheesman, Jennilea Courtney, Sabrina Hamer, University of Ottawa; Paul Barré, MD, Jeffrey Golden, Royal Victoria Hospital; Brendan Barrett, MD, Elizabeth Langille, Sandra Adams, Janet Morgan, Eastern Regional Health Authority, Health Sciences Centre; Catherine Clase, MD, Cathy Moreau, St Joseph's Hospital; Susan Cooper, MD, Brian Forzley, MD, Susan Caron, Shauna Granger, Penticton Regional Hospital; Serge Cournoyer, MD, Lorraine Menard, Michèle Roy, Hélène Skidmore, Dolores Beaudry, Charles Le Moyne Hospital; Janis Dionne, MD, Josephine Chow, Valla Sahraei, BC Children's Hospital; Sandra Donnelly, MD, Niki Dacouris, Rosa Marticorena, St. Michael's Hospital; Brenda Hemmelgarn, MD, Sharon Gulewich, Troy Hamilton, Foothills Hospital; Paul Keown, MD, Nadia Zalunardo, MD, Daniel Rogers, Reena Tut, Matthew Paquette, Vancouver General Hospital; Adeera Levin, Taylor Perry, Zainab Sheriff, Nancy Ferguson, Helen Chiu, Ilinka Zrno, Kathleen Carlson, Lina Sioson, St. Paul's Hospital; Charmaine Lok, MD, Michelle Cross, University Health Network; François Madore, MD, Manon Maltais, Hôpital du Sacré-Cœur; Louise Moist, MD, Kerri Gallo, Sarah Langford, Leah Slamen, London Health Science Centre-Victoria Campus; Norman Muirhead, MD, Mary Jeanne Edgar, Taylor Gray, Cameron Edgar, Karen Groeneweg, Eileen McKinnon, Erin McRae, London Health Science Centre-University Campus; Bharat Nathoo, MD, Kimmy Lau, York Central; Malvinder Parmar, MD, Sylvie Gelinas, Timmins \& District Hospital; Martine Leblanc, MD, Lucie Lépine, Maisonneuve-Rosemont Hôpital; Claudio Rigatto, MD, Dolores Friesen, Marla Penner, St. Boniface Hospital; Steven Soroka, MD, Susan Fleet, Jeanette Squires, QEII Health Sciences Centre; Siva Thanamayooran, MD, Michael Binder, Christine Hines, Brenda McNeil, Cape Breton District Health Authority; Sheldon Tobe, MD, Mary Chessman, Nancy Perkins, Martha Agelopoulos, Stacey Knox, Sunnybrook Hospital; Marcello Tonelli, Susan Szigety, Dawn Opgenorth, University of Alberta; Karen Yeates, MD, Karen Mahoney, Kingston General Hospital.

\section{Statement of Ethics}

All participants provided informed consent. The protocol was approved by the University of British Columbia Research Ethics Board. 


\section{Disclosure Statement}

M.W.: has served as a consultant or received honoraria from Akebia, Amag, Amgen, Ardelyx, DiaSorin, Keryx, and Sanofi and received grant support from Shire. A.L.: has served as a consultant or received honoraria from Otsuka, Boehringer Ingelheim, and Janssen, and received grant support from Amgen and Otsuka.

\section{Funding Sources}

We gratefully acknowledge the financial support from DiaSorin Inc., through an unrestricted grant and for providing all reagents and laboratory analysis for the Bone \& Mineral Bio- marker panel. We also acknowledge the financial support obtained from Janssen Canada for the original CanPREDDICT study.

\section{Author Contributions}

A.L., O.D., and M.C.: determined the concept, rationale, design logistics, and analysis. M.W., M.C., A.L., M.T., and O.D.: developed and wrote the manuscript. M.W., C.Z., and F.B.: provided constructive criticism and edits of the drafts of the manuscripts. O.D.: was responsible for all analyses and presentation of the materials in the manuscript. All contributed to the final product.

\section{References}

1 Isakova T, Gutiérrez OM, Patel NM, Andress DL, Wolf M, Levin A. Vitamin D deficiency, inflammation, and albuminuria in chronic kidney disease: complex interactions. J Ren Nutr. 2011 Jul;21(4):295-302.

2 Levin A, Le Barbier M, Er L, Andress D, Sigrist MK, Djurdjev O. Incident isolated 1,25(OH) (2) $\mathrm{D}(3)$ deficiency is more common than 25(OH)D deficiency in CKD. J Nephrol. 2012 Mar-Apr;25(2):204-10.

3 Wolf M. Update on fibroblast growth factor 23 in chronic kidney disease. Kidney Int. 2012 Oct;82(7):737-47.

4 Gutierrez O, Isakova T, Rhee E, Shah A, Holmes J, Collerone G, et al. Fibroblast growth factor-23 mitigates hyperphosphatemia but accentuates calcitriol deficiency in chronic kidney disease. J Am Soc Nephrol. 2005 Jul;16(7):2205-15.

5 Quarles LD. The bone and beyond: 'Dem bones' are made for more than walking. Nat Med. 2011 Apr;17(4):428-30.

6 Kendrick J, Cheung AK, Kaufman JS, Greene T, Roberts WL, Smits G, et al.; HOST Investigators. FGF-23 associates with death, cardiovascular events, and initiation of chronic dialysis. J Am Soc Nephrol. 2011 Oct;22(10):1913-22.

7 Isakova T, Xie H, Yang W, Xie D, Anderson AH, Scialla J, et al.; Chronic Renal Insufficiency Cohort (CRIC) Study Group. Fibroblast growth factor 23 and risks of mortality and end-stage renal disease in patients with chronic kidney disease. JAMA. 2011 Jun;305(23): 2432-9.

8 Block GA, Klassen PS, Lazarus JM, Ofsthun N, Lowrie EG, Chertow GM. Mineral metabolism, mortality, and morbidity in maintenance hemodialysis. J Am Soc Nephrol. 2004 Aug;15(8):2208-18.

9 Ketteler M, Block GA, Evenepoel P, Fukagawa $\mathrm{M}$, Herzog CA, McCann L, et al. Executive summary of the 2017 KDIGO Chronic Kidney Disease-Mineral and Bone Disorder (CKD-MBD) Guideline Update: what's changed and why it matters. Kidney Int. 2017 Jul;92(1):26-36.
10 Levin A, Rigatto C, Brendan B, Madore F, Muirhead N, Holmes D, et al.; CanPREDDICT investigators. Cohort profile: canadian study of prediction of death, dialysis and interim cardiovascular events (CanPREDDICT). BMC Nephrol. 2013 Jun;14(1):121.

11 Valcour A, Zierold C, Blocki FA, Hawkins DM, Martin KJ, Rao SD, et al. Trueness, precision and stability of the LIAISON 1-84 parathyroid hormone (PTH) third-generation assay: comparison to existing intact PTH assays. Clin Chem Lab Med. 2018 Aug;56(9):147682.

12 Cavalier E, Plebani M, Delanaye P, Souberbielle JC. Considerations in parathyroid hormone testing. Clin Chem Lab Med. 2015 Nov; 53(12):1913-9.

13 Souberbielle JC, Boutten A, Carlier MC, Chevenne D, Coumaros G, Lawson-Body E, et al. Inter-method variability in PTH measurement: implication for the care of CKD patients. Kidney Int. 2006 Jul;70(2):345-50.

14 Sturgeon CM, Sprague S, Almond A, Cavalier E, Fraser WD, Algeciras-Schimnich A, et al.; IFCC Working Group for PTH. Perspective and priorities for improvement of parathyroid hormone (PTH) measurement - A view from the IFCC Working Group for PTH. Clin Chim Acta. 2017 Apr;467:42-7.

15 Souberbielle JC, Prié D, Piketty ML, Rothenbuhler A, Delanaye P, Chanson P, et al. Evaluation of a New Fully Automated Assay for Plasma Intact FGF23. Calcif Tissue Int. 2017 Nov;101(5):510-8.

16 Levey AS, Stevens LA, Schmid CH, Zhang YL, Castro AF 3rd, Feldman HI, et al.; CKD-EPI (Chronic Kidney Disease Epidemiology Collaboration). A new equation to estimate glomerular filtration rate. Ann Intern Med. 2009 May;150(9):604-12.

17 Tangri N, Stevens LA, Griffith J, Tighiouart $\mathrm{H}$, Djurdjev O, Naimark D, et al. A predictive model for progression of chronic kidney disease to kidney failure. JAMA. 2011 Apr; 305(15):1553-9.
18 Contal C, O'Quigley J. An application of changepoint methods in studying the effect of age on survival in breast cancer. Comput Stat Data Anal. 1999;30(3):253-70.

19 Williams BA, Mandrekar JN, Mandrekar SJ, Cha SS, Furth AF. Technical Report Series \#79: Finding Optimal Cutpoints for Continuous Covariates with Binary and Time-toEvent Outcomes. Department of Health Science Research, Mayo Clinic, Rochester; 2006.

20 Miller R, Siegmund D. Maximally Selected Chi-Square Statistics. Biometrics. 1982;38(4): 1011-6.

21 Chambless LE, Cummiskey CP, Cui G. Several methods to assess improvement in risk prediction models: extension to survival analysis. Stat Med. 2011 Jan;30(1):22-38.

22 Chambless LE, Diao G. Estimation of timedependent area under the ROC curve for long-term risk prediction. Stat Med. 2006 Oct;25(20):3474-86.

23 Pencina MJ, D’Agostino RB Sr, D’Agostino RB Jr, Vasan RS. Evaluating the added predictive ability of a new marker: from area under the ROC curve to reclassification and beyond. Stat Med. 2008 Jan;27(2):157-72.

24 Pencina MJ, D’Agostino RB Sr, Steyerberg EW. Extensions of net reclassification improvement calculations to measure usefulness of new biomarkers. Stat Med. 2011 Jan;30(1): $11-21$.

25 Floege J, Kim J, Ireland E, Chazot C, Drueke T, de Francisco A, et al.; ARO Investigators. Serum iPTH, calcium and phosphate, and the risk of mortality in a European haemodialysis population. Nephrol Dial Transplant. 2011 Jun;26(6): 1948-55.

26 Covic A, Kothawala P, Bernal M, Robbins S, Chalian A, Goldsmith D. Systematic review of the evidence underlying the association between mineral metabolism disturbances and risk of all-cause mortality, cardiovascular mortality and cardiovascular events in chronic kidney disease. Nephrol Dial Transplant. 2009 May;24(5):1506-23. 
27 Marmorino F, Salvatore L, Barbara C, Allegrini G, Antonuzzo L, Masi G, et al. Serum LDH predicts benefit from bevacizumab beyond progression in metastatic colorectal cancer. Br J Cancer. 2017 Jan;116(3):318-23.

28 Palatini P, Reboldi G, Beilin LJ, Casiglia E, Eguchi K, Imai Y, et al. Added predictive value of night-time blood pressure variability for cardiovascular events and mortality: the Ambulatory Blood Pressure-International Study. Hypertension. 2014 Sep;64(3):48793.

29 Abajo M, Betriu À, Arroyo D, Gracia M, Del Pino MD, Martínez I, et al.; Investigators of the NEFRONA study. Mineral metabolism factors predict accelerated progression of common carotid intima-media thickness in chronic kidney disease: the NEFRONA study. Nephrol Dial Transplant. 2017 Nov;32(11): 1882-91.

30 Faul C, Amaral AP, Oskouei B, Hu MC, Sloan A, Isakova T, et al. FGF23 induces left ventricular hypertrophy. J Clin Invest. 2011 Nov; 121(11):4393-408.

31 Block GA, Wheeler DC, Persky MS, Kestenbaum B, Ketteler M, Spiegel DM, et al. Effects of phosphate binders in moderate CKD. J Am Soc Nephrol. 2012 Aug;23(8):1407-15.

32 Isakova T, Barchi-Chung A, Enfield G, Smith $\mathrm{K}$, Vargas G, Houston J, et al. Effects of dietary phosphate restriction and phosphate binders on FGF23 levels in CKD. Clin J Am Soc Nephrol. 2013 Jun;8(6):1009-18.

33 Altman DG. Categorising continuous variables. Br J Cancer. 1991 Nov;64(5):975.

34 Courdi A, Héry M, Chauvel P, Gioanni J, Namer M, Demard F. Prognostic value of continuous variables in breast cancer and head and neck cancer. Dependence on the cut-off level. Br J Cancer. 1988 Jul;58(1):88-90.

35 Mazumdar M, Glassman JR. Categorizing a prognostic variable: review of methods, code for easy implementation and applications to decision-making about cancer treatments. Stat Med. 2000 Jan;19(1):113-32. 